\title{
Factors associated with the number of clinical pharmacy recommendations: findings from an observational study in geriatric inpatients
}

\author{
Lorenz Van Der Linden $\left(\mathbb{D}^{\mathrm{a}, \mathrm{b}}\right.$, Julie Hias $\mathbb{D}^{\mathrm{a}}{ }^{\text {, }}$ Karolien Walgraeve ${ }^{\mathrm{a}}$, Silke Loyens ${ }^{\mathrm{c}}$, Johan Flamaing ${ }^{\mathrm{d}, \mathrm{e}}$, Isabel Spriet ${ }^{\mathrm{a}, \mathrm{b}}$ \\ and Jos Tournoyd,e \\ aPharmacy Department, University Hospitals Leuven, Leuven, Belgium; 'bepartment of Pharmaceutical and Pharmacological Sciences, KU \\ Leuven, Leuven, Belgium; 'Faculty Of Biomedical Sciences, KU Leuven, Leuven, Belgium; ${ }^{\mathrm{d} D e p a r t m e n t}$ of Geriatric Medicine, University \\ Hospitals Leuven, Leuven, Belgium; 'Department of Chronic Diseases, Metabolism and Ageing, KU Leuven, Leuven, Belgium
}

\section{ABSTRACT}

Objectives: Drug-related problems are prevalent in older inpatients and can be reduced by providing clinical pharmacy $(C P)$ services. Details concerning implementation in clinical practice are frequently lacking. The aim was to describe the output of one such CP program and to identify factors associated with CP recommendations.

Methods: A CP program was installed at three acute geriatric wards in a teaching hospital. A convenience sample was collected, consisting of inpatients who received a CP consultation at discharge. Medical conditions, patient demographics, and drug use were evaluated retrospectively. Number and type of the $\mathrm{CP}$ recommendations were determined. A Poisson regression analysis was performed to determine factors associated with the number of $\mathrm{CP}$ recommendations.

Results: A cohort of 524 patients, aged 85 (interquartile range (IQR): 82-89) years was included. On admission, 10.31 (standard deviation: 4.49) drugs were taken. Three (IQR: 2-4) CP recommendations were provided per patient, of which $70.2 \%$ targeted drug discontinuation. A model was derived, containing the following factors: number of drugs on admission (incidence rate ratio (IRR): 1.063; 95\% confidence interval (Cl): 1.052-1.074), number of previous contacts with the geriatric department (IRR: $0.869 ; 95 \% \mathrm{Cl}$ : 0.816-0.926), presence of left-ventricular dysfunction (IRR: $1.179,95 \% \mathrm{Cl}: 1.023-1.360$ ), the number of new drugs (IRR: $1.046 ; 95 \% \mathrm{Cl}: 1.021-1.071$ ) and use of colecalciferol (IRR: 1.22; $95 \% \mathrm{Cl}$ : 1.088-1.367).

Conclusions: Five factors were associated with the number of $\mathrm{CP}$ recommendations at discharge. This could allow for further patient stratification to increase the efficiency of the $C P$ program.

\section{KEYWORDS}

Clinical pharmacy; geriatrics; inpatients; risk stratification; polypharmacy

\section{Introduction}

Clinical pharmacy $(C P)$ services have seen an increased uptake over the past decades [1-4]. CP research has mainly targeted patients at increased risk for drugrelated problems (DRP). As DRP frequently occur in older inpatients, this group has regularly been targeted in $C P$ investigations. $C P$ interventions have already been shown to decrease inappropriate prescribing in older inpatients [3-5]. Particularly, the Integrated Medicines Management (IMM) model and its Swedish analogue, the Lund IMM model, deserve more attention [6-8]. The LIMM model entails the systematic provision of pharmaceutical care on admission, during hospital stay and at discharge. This approach has been associated with fewer potentially inappropriate medications (PIMs), adverse drug reactions and hospital visits. The overall evidence base in support of inpatient CP services is growing $[5,9,10]$.

In the University Hospitals of Leuven hospital pharmacists have been allocated sparingly to provide CP services. One of those activities concerned a CP program (0.8 full-time equivalent (FTE)) for 78 acute geriatric beds [11,12]. Over a three-year period, a consensus was reached among the care team to allocate project time to perform medication reviews before discharge. The aim of the $C P$ program was to ensure optimal drug therapy during care transitions, strengthened by the recent findings of Pellegrin et al. and Ravn-Nielsen et al. [10,13]. Both authors showed that hospital pharmacists were able to reduce the number of unplanned hospital visits $[10,13]$.

Some pertinent questions remain however on the benefits of CP services in daily clinical practice. It is unclear how study findings should be implemented as the external validity of investigations might be limited [14]. For example, in Belgium, only one hospital pharmacist is budgeted per 150 beds, with limited additional governmental funding to support extra CP services (0.25 FTE per 250 beds) [15]. The aim of this study was therefore to document the output of the current CP program implemented on the acute geriatric care wards, and to determine factors associated 
with the number of provided recommendations at discharge.

\section{Methods}

\section{Study design and setting}

A retrospective study was performed at a 2000-bed teaching hospital, the University Hospitals Leuven, Leuven, Belgium. Patient data were retrieved from a sample of electronic patient files in a period ranging from April 2016 until December 2016. Approval for the study was obtained from the Supervisory Committee on Medical Ethics of the KU Leuven, Leuven, Belgium.

\section{Clinical pharmacy services}

A ward-based CP program was established at three acute geriatric care wards in October 2014. Hospital management provided support for a $0.8 \mathrm{FTE}$, during which drug therapies were consecutively reviewed. No predefined selection criteria were used in the CP program.

Clinical pharmacists performed a structured medication reconciliation upon request of the physicians, performed medication reviews and generated patientfriendly medication lists at discharge $[11,16]$. An implicit medication review approach was followed, taking into account patient preferences, time to benefit and estimated life expectancy [17-19]. All recommendations were communicated directly to the treating physician and registered in a structured form that was part of the electronic health record (EHR). Accepted recommendations were adopted into the discharge letter.

\section{Participants}

Patients were included in the analysis, if admitted to the acute geriatric wards and if the pharmacist performed a medication review prior to discharge. If the pharmacist performed a medication review on admission or during hospital stay, the patient was excluded from analysis, to guarantee a temporal relation between the factors and the number of recommendations at discharge used in the regression analysis.

\section{Data collection}

Patient data were retrieved independently by two research students. Collected data were subsequently audited by a third researcher (SL). The EHR included the CP forms, medical files, the electronic prescriptions, the laboratory data as well as the nursing notes. Coded information (e.g. International Classification of Diseases classification (ICD)) was not available at the time of data collection due to the lag time (i.e. about one year) experienced by the hospital's administration dept.
The structured CP form was used to gather information on the number and type of recommendations, the medications for which recommendations were made and the acceptance rate by the attending physician.

Following data were collected during hospital stay: gender, age, length of stay, living situation before and after hospitalization, do-not-resuscitate (DNR) code, Mini-Mental State Examination (MMSE) score, number of drugs on admission, at discharge and initiated during hospital stay, risk of falling (mentioned as such in the EHR or if a fall in the previous year had been documented), the (probable) diagnosis on admission and whether a fall was an admission diagnosis [20]. Drug information was evaluated at the Anatomical Therapeutic Chemical (ATC) classification 5 level and included all drugs that the patient received. The diagnosis on admission was categorized as cardiovascular, infectious, pulmonary, renal, gastrointestinal, psychiatric, neurological, traumatic or skeletal, related to a decline of the patient's general condition, due to a fall, a malignancy, or due to social issues. Furthermore, it was verified whether the patient was admitted through the emergency department (ED), whether the patient was transferred from another hospital or ward within the hospital or via a preceding intensive care unit (ICU) admission. Also, the number of consultations by other medical specialties during the index stay was determined as it might have been a proxy for the disease severity of the patient.

Data on hospitalizations during the previous year were collected, including the number of hospitalizations, the number of ED visits and ICU stays. The total number of previous contacts with the geriatric department (dept.) was determined as well, without further discriminating between type of contact (e.g. hospital admission, consultation, ambulatory clinic). In addition, information was collected on the following measures that occurred during the 30 days prior to the index hospital stay: total number of overall hospitalizations and hospitalizations to the acute geriatric wards and also the number of ED-visits. The numbers of consultations by medical specialties and by the hospital pharmacy team, before the hospital stay, were also gathered.

Information on medical conditions was obtained from the discharge letter and was categorized according to organ system. Presence of these conditions was stratified as being not present at all, a previously known diagnosis (i.e. before index admission), a new diagnosis, or as timing uncertain. Further details on collected conditions, laboratory values and nursing notes can be consulted in the Online Appendix.

\section{Statistical analysis}

Normality was determined by visual inspection of the histograms. Parametric continuous variables were expressed as means and standard deviations (SD), 
non-parametric variables as medians and interquartile ranges (IQR: Q1-Q3). Categorical data were summarized as counts and percentages.

A Kruskal-Wallis analysis was used to compare the number of recommendations between months to determine whether a teaching effect occurred. A paired t-test was used to compare the number of drugs on admission and at discharge.

Poisson regression analysis was performed to identify factors associated with the dependent outcome of the number of recommendations at discharge. First, a univariable Poisson regression analysis was performed. Owing to the explorative nature of the study, all patient characteristics were tested as detailed in the section on Data Collection. Second, factors were selected for multivariable Poisson regression analysis, based on statistical significance $(p<0.05)$ shown in the univariable analysis and on plausible association according to the researchers. Third, a backward stepwise approach was followed to reach a parsimonious model. The aim of this backward stepwise approach was to explore the association of a multitude of factors on the number of recommendations. A lower Akaike information criterion (AIC) value was preferred in model selection.

Predicted and observed counts were dichotomized, according to predefined thresholds (i.e. $\geq 3$ to $\geq 5$ ) for the number of pharmacotherapeutic recommendations. These thresholds were selected to identify patients who were more likely to receive at least the median number of three recommendations. To estimate the diagnostic performance of the regression model, the sensitivity, specificity, positive and negative predictive values were calculated as well as the positive and negative likelihood ratios $(\mathrm{LH})$ at several predefined thresholds. The alert rate was calculated as the number of patients identified by using the model at a specified threshold. A higher positive likelihood ratio (>5.0) was preferred in evaluating the predictive performance in order to avoid false positives [21].

Data analysis was performed with IBM Statistical Package Version 25.0 (Armonk, NY: IBM Corp.). Tests were considered statistically significant if the twosided $\mathrm{p}$-value was $<0.05$.

\section{Results}

\section{Patient characteristics}

From 25 April 2016 until 2 December 2016, 1215 patients were admitted to the geriatric wards, of whom 726 (59.8\%) received at least 1 CP consultation during their hospital stay. In 698 patients a cause for admission was documented in the medical file. A majority of admissions (69.3\%) was explained by five conditions: infectious diseases (20.8\%), pulmonary conditions (13.2\%), a fall (12.8\%), and cardiovascular (11.6\%) or gastrointestinal conditions (11.0\%).
The final study population consisted of 524 patients, who had received at least 1 medication review at the moment of hospital discharge (43.1\%). On average, patients were 85 years old and $66 \%$ of them were admitted from home. Cardiovascular disease burden was high, with high prevalences of valvular dysfunction $(65.8 \%)$, atrial fibrillation (44.5\%) and heart failure (37.6\%). More information concerning demographics has been summarized in Table 1. Additional information can be retrieved in the Online Appendix (Tables 4 to 9).

\section{Drug use}

Upon admission, 414 different ATC classes and 5345 drugs were taken by 519 patients; 1 patient took no drugs on admission; contradictory information was found in 4 patients. The drug classes most commonly used were cardiovascular medications (ATC B + C) $(42 \%)$, followed by alimentary $(25 \%)$ and central nervous system medications (16\%). The following five drugs were used most frequently upon admission: paracetamol (46.0\%), bisoprolol (40.5\%), combination of calcium and vitamin D (36.3\%), low-dose acetylsalicylic acid (35.5\%) and pantoprazole (35.5\%).

During hospital stay, 1546 additional medications were started in 468 patients and at discharge 5203

Table 1. Demographics and clinical characteristics.

\begin{tabular}{|c|c|}
\hline \multicolumn{2}{|l|}{ Demographics $(n=524)$} \\
\hline Age (years), median (IQR) & $85(82,89)$ \\
\hline Female, $\%$ & $57.6 \%$ \\
\hline Length of stay (days), median (IQR) & $12(9,18)$ \\
\hline Weight $(\mathrm{kg})$, median $(\mathrm{IQR})(\mathrm{n}=500)$ & $66(56,76)$ \\
\hline MMSE (on 30), median (IQR) $(n=372)$ & $22(18,26)$ \\
\hline DNR 0, DNR 1 or DNR $2, \%$ & $\begin{array}{c}48.5 \%, 12.6 \% \text { or } \\
38.9 \%\end{array}$ \\
\hline $\mathrm{SCr}(\mathrm{mg} / \mathrm{dl})$, median (IQR) & $1.27(0.93,1.75)$ \\
\hline $\mathrm{CrCl}(\mathrm{CG}, \mathrm{ml} / \mathrm{min})$, median (IQR) & $36.1(25.3,49.5)$ \\
\hline eGFR (CKD-EPI, ml/min/1.73m²), median (IQR) & $45.5(30.0,61.1)$ \\
\hline Number of drugs on admission, mean (SD) & $10.31(4.49)$ \\
\hline \multicolumn{2}{|l|}{ Clinical Characteristics, $\%^{\mathrm{a}}$} \\
\hline Valvular dysfunction & $65.8 \%$ \\
\hline Arterial hypertension & $55.0 \%$ \\
\hline Atrial fibrillation & $44.5 \%$ \\
\hline Heart failure & $37.6 \%$ \\
\hline Diabetes mellitus & $30.0 \%$ \\
\hline Hypercholesterolemia & $28.1 \%$ \\
\hline Osteoporosis & $27.5 \%$ \\
\hline Delirium or psychosis & $24.8 \%$ \\
\hline $\begin{array}{l}\text { Cerebrovascular accident of transient ischemic } \\
\text { attack }\end{array}$ & $19.8 \%$ \\
\hline Recurrent falling & $18.5 \%$ \\
\hline COPD & $18.5 \%$ \\
\hline Cancer history ( $>5$ years) & $17.7 \%$ \\
\hline Dyspepsia or esophagitis & $17.0 \%$ \\
\hline Percutaneous coronary intervention & $16.6 \%$ \\
\hline Severe aortic stenosis $\left(<1 \mathrm{~cm}^{2}\right)$ & $16.2 \%$ \\
\hline Gastric or duodenal ulcers & $15.6 \%$ \\
\hline Ejection fraction $<40 \%$ & $12.0 \%$ \\
\hline Active cancer & $10.5 \%$ \\
\hline
\end{tabular}

${ }^{\mathrm{a} C l i n i c a l}$ conditions with a prevalence above $10 \%$ are depicted.

Abbreviations: IQR (interquartile range), SD (standard deviation), MMSE (Mini Mental State Examination), DNR 0 (no do-not-resuscitate code), DNR 1 (do no reanimate), DNR 2 (life-prolonging interventions are withheld), $\mathrm{SCr}$ (serum creatinine), $\mathrm{CrCl}$ (creatinine clearance), CG (Cockcroft-Gault) [34], eGFR (estimated glomerular filtration rate), CKDEPI (Chronic Kidney Disease - Epidemiology Collaboration) [35], COPD (Chronic Obstructive Pulmonary Disease). 
medications were prescribed to 516 patients. On average, 3 (IQR: 1, 4) drugs were initiated per patient. The most frequently initiated medications were: calcium and vitamin D (12.8\%), a macrogol laxative (9.4\%), paracetamol (6.2\%), bumetanide (2.9\%) and pantoprazole $(2.7 \%)$.

Hospital stay was not associated with a reduction in the total number of medications (admission: 10.31 (SD 4.49) vs. discharge: 10.10 (SD 3.73), $\mathrm{P}=0.138$ ).

\section{Clinical pharmacy recommendations}

A total number of $1582 \mathrm{CP}$ recommendations was provided to 524 patients, with a median number of 3 (IQR: 2-4) per patient. No recommendations were recorded in eleven patients. Approximately a fifth of the cohort (19.7\%) received at least five CP recommendations. The acceptance rate of the individual recommendations by the treating physicians was $87.2 \%$.

The $C P$ recommendations mainly concerned the following drug classes: cardiovascular (28.7\%), psychotropic (16.6\%) and gastrointestinal drugs (11.7\%), as well as vitamins $(11.6 \%)$ and drugs for pain relief or anti-inflammatory effects (7.2\%). Drug discontinuation was recommended in $70.2 \%$ of the patients, followed by a dose or frequency adjustment in $45.4 \%$ and drug initiation in $30.2 \%$.

No significant differences were observed in the number of CP recommendations between the studied months (April to December 2016) $(p=0.149)$.

\section{Factors associated with the number of CP recommendations at discharge}

Twenty-seven individual variables were found to be significantly correlated with the number of CP recommendations at discharge, using a univariable Poisson regression analysis. Detailed results of the Poisson regression have been added to the Online Appendix (Table 10).

An explorative model was derived and was based on the following independent factors: number of previous contacts with the geriatric dept. (mean per patient: 0.49 (SD 0.943)), number of drugs on admission (10.3 (SD 4.7)), number of initiated drugs during hospital stay (2.95 (SD 2.06)), the use of colecalciferol on admission (20.8\%) and the presence of left ventricular dysfunction, characterized by an ejection fraction of less than $40 \%$ (11.9\%).

Model characteristics, including the incidence rate ratio's (IRR) for the individual factors and the derived regression equations, have been summarized in Table 2. More than $95 \%$ of the standardized deviance residuals were situated between values of -2 and 2 . No multicollinearity was observed among the continuous variables. Furthermore, visual analysis of the boxplot showed a moderate correlation between the predicted
Table 2. Model characteristics and variables.

\begin{tabular}{|c|c|c|}
\hline \multicolumn{3}{|l|}{ Model Characteristics } \\
\hline Number of patients & \multicolumn{2}{|l|}{519} \\
\hline Deviance/df & \multicolumn{2}{|l|}{0.940} \\
\hline $\mathrm{AIC}$ & \multicolumn{2}{|c|}{1943.745} \\
\hline Likelihood Ratio Chi Square & \multicolumn{2}{|c|}{$P<0.001$} \\
\hline RMSE & \multicolumn{2}{|l|}{1.738} \\
\hline Variables & IRR (95\% Cl) & P-value \\
\hline $\begin{array}{l}\text { Number of drugs on } \\
\text { admission }\end{array}$ & $1.063(1.052-1.074)$ & $<0.001$ \\
\hline$E F<40 \%$ & $1.179(1.023-1.360)$ & 0.023 \\
\hline $\begin{array}{l}\text { Number of previous contacts } \\
\text { with the geriatric dept. }\end{array}$ & $0.869(0.816-0.926)$ & $<0.001$ \\
\hline $\begin{array}{l}\text { Number of initiated drugs } \\
\text { during hospital stay }\end{array}$ & $1.046(1.021-1.071)$ & $<0.001$ \\
\hline $\begin{array}{l}\text { Use of colecalciferol on } \\
\text { admission }\end{array}$ & $1.220(1.088-1.367)$ & 0.001 \\
\hline $\begin{array}{l}\text { Regression equation: } \\
\text { number of predicted } \\
\text { recommendations at } \\
\text { discharge = }\end{array}$ & \multicolumn{2}{|c|}{$\begin{array}{l}\text { Exp }\left(0.306+0.165^{*} \mathrm{EF}<40 \%+\right. \\
0.061^{*} \text { number of drugs on admission }+ \\
0.45^{*} \text { newly initiated drugs }+0.199^{*} \text { use } \\
\text { of colecalciferol on admission }-0.140^{*} \\
\text { number of previous contacts with the } \\
\text { geriatric dept.) }\end{array}$} \\
\hline
\end{tabular}

Abbreviations: AIC (Akaike Information Criterion), RSME (root-meansquare error), IRR (incidence rate ratio), EF (ejection fraction), Cl (confidence interval), df (degrees of freedom).

and observed number of recommendations prior to discharge, as depicted in Figure 1.

Predictive characteristics were estimated and have been summarized in Table 3. A higher positive likelihood ratio was observed as the threshold for the number of recommendations increased, which coincided with higher specificities and negative predictive values.

\section{Discussion}

To the best of our knowledge, this is the first report on the output of a CP program, that includes an explorative analysis on variables associated with the number of recommendations prior to discharge. Feras et al. described several algorithms to identify high-risk patients, yet pharmacy staffing and not the actual number of provided recommendations were included in their analysis [22].

Five factors were identified and a Poisson regression model was selected based on the lowest AIC. The explorative model consisted of the following factors: number of drugs on admission $(6.3 \%$ increase in the number of $\mathrm{CP}$ recommendations per drug), number of newly initiated drugs during hospital stay $(4.6 \%$ increase per drug), the number of previous contacts with the geriatric dept. (13.1\% decrease per contact), the use of colecalciferol on admission (22\% increase in the number of $\mathrm{CP}$ recommendations) and the presence of an ejection fraction less than $40 \%$ (17.9\% increase in the number of $\mathrm{CP}$ recommendations). A pragmatic approach could be preferred by first selecting patients with no previous contacts with the geriatrics department, as this concerned the single largest factor, in terms of its beta coefficient.

In a tentative analysis, the final model pointed toward a good predictive ability if applied as a screening test. The 


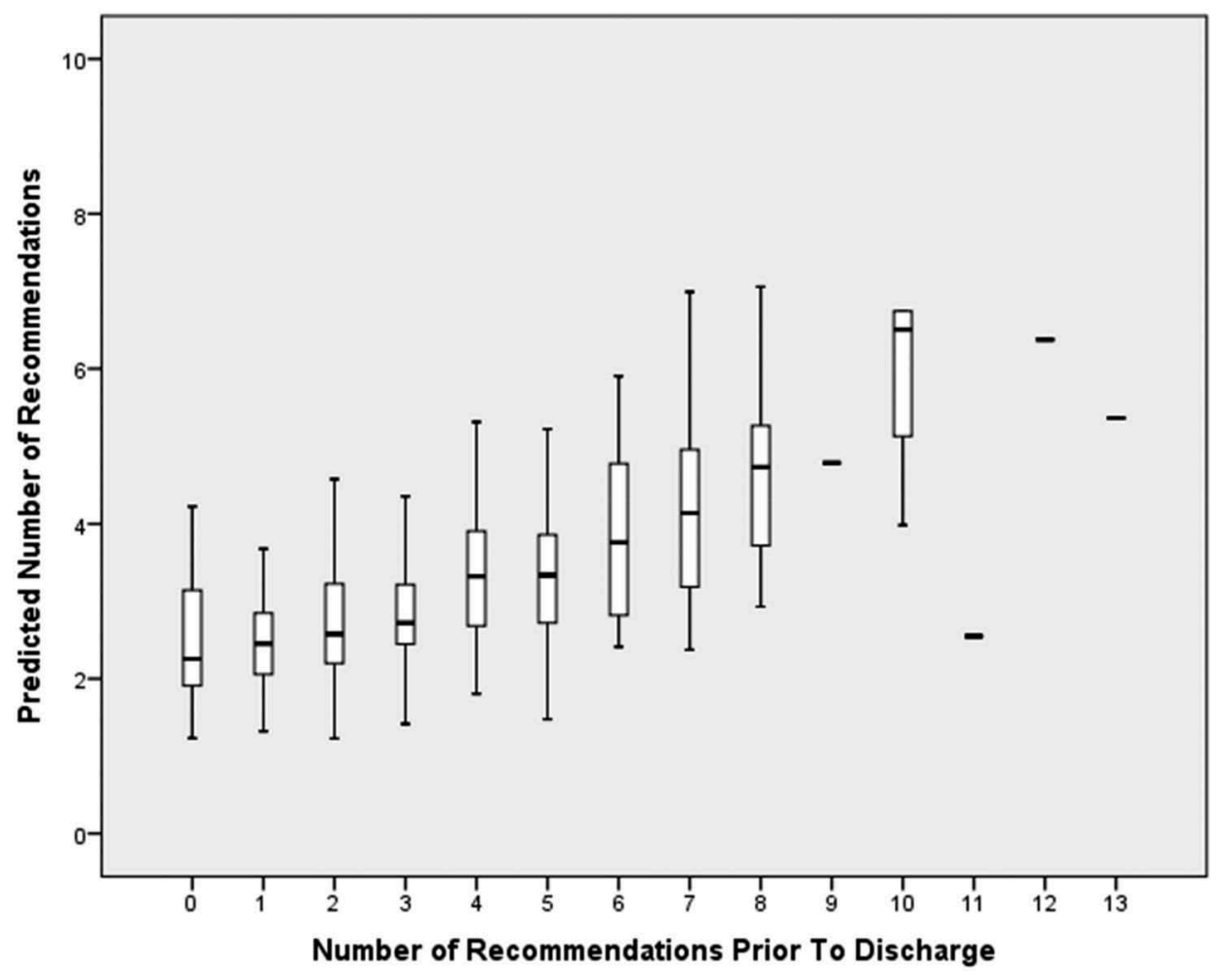

Figure 1. The number of estimated recommendations as a function of the number of observed recommendations.

Box and whisker plots of the final exploratory model are shown with the estimated number of pharmacotherapeutic recommendations as a function of the observed number of recommendations.

Table 3. Model predictive ability according to thresholds for the number of recommendations.

\begin{tabular}{|c|c|c|c|c|c|c|c|}
\hline Threshold & Sensitivity & Specificity & PPV & NPV & $\mathrm{LH}+$ & LH- & $\begin{array}{l}\text { Alert } \\
\text { rate }\end{array}$ \\
\hline$\geq 3$ & $79.1 \%(74.2 \%-84.0 \%)$ & $49.2 \%(43.1 \%-55.3 \%)$ & $61.5 \%(56.4 \%-66.7 \%)$ & $69.6 \%(62.9 \%-76.3 \%)$ & $1.557(1.360-1.784)$ & $0.425(0.326-0.554)$ & $65.13 \%$ \\
\hline$\geq 4$ & $51.8 \%(44.2 \%-59.3 \%)$ & $86.3 \%(82.7 \%-89.9 \%)$ & $64.4 \%(56.4 \%-72.5 \%)$ & $78.9 \%(74.8 \%-83.0 \%)$ & $3.787(2.804-5.115)$ & $0.559(0.475-0.657)$ & $26.01 \%$ \\
\hline$\geq 5$ & $29.1 \%(20.4 \%-37.9 \%)$ & $96.9 \%(95.2 \%-98.5 \%)$ & $69.8 \%(56.0 \%-83.5 \%)$ & $84.7 \%(81.4 \%-87.9 \%)$ & $9.320(5.0544-17.223)$ & $0.732(0.646-0.829)$ & $8.29 \%$ \\
\hline
\end{tabular}

model showed a $L R+$ of almost 10 , indicating a moderate to strong likelihood of identifying patients with at least five drug recommendations [21]. The lower sensitivity was accepted at higher thresholds, as our findings confirmed that there were almost no patients $(11 / 524,2.1 \%)$ at discharge, in whom no recommendations were provided. Furthermore, clinical demand exceeded the limits of the program, signified by a $59.8 \%$ capture rate, highlighting the need for risk stratification (i.e. patient selection by applying a model with a high LR+).

The profile of our patient population and by extension, that of the subset of patients identified by the count regression analysis, mostly corresponded with that of the geriatric, polymedicated and complex patient profile. Most associative factors have already been described and our study findings seem to confirm previous reports. For example, the total number of drugs (i.e. number of drugs on admission and the number of newly initiated drugs during hospital stay) has been identified as a risk factor for adverse (drug) events during hospital stay and afterwards [22,23].
Presence of an ejection fraction $<40 \%$ identifies patients who are likely to take multiple drugs, but also who should avoid certain therapies (e.g. verapamil) and in whom drug initiation and up-titration (e.g. of beta-blockers) could still be of clinical benefit, even in high age as time to benefit is short (i.e. months) $[24,25]$. The number of previous contacts with the geriatric dept. was inversely correlated with the number of CP recommendations, which can be explained by patients having already been reviewed by a multidisciplinary team during previous hospital admissions. Use of colecalciferol could have indirectly identified patients with a higher frailty, or renal impairment, perhaps necessitating the use of dual calcium and vitamin D treatment as well as the correct administration of antiresorptive agents.

An average of three drug-related recommendations per patient was provided, which remained stable during the study period. This could potentially be explained by the inherent added value of the clinical pharmacists within the multidisciplinary team $[3,4]$. Conversely, it 
could also indicate a suboptimal teaching effect and the lack of academic detailing. The number and acceptance rate of the $\mathrm{CP}$ recommendations were higher than those observed in recent comparable CP programs in the Flanders region $[26,27]$. In contrast, the acceptance rate was comparable to our own previous experiences [11]. The higher acceptance rates might be explained by the ward-based presence of clinical pharmacists [28]. We hypothesize that the teaching hospital environment with many physicians-in-training might have only had a minor impact on the acceptance rate, as evidenced by Ryan et al. [29]. In many hospitals, including nonteaching hospitals, junior physicians will write the majority of prescriptions.

Deprescribing was recommended in a majority (70.2\%) of patients. Deprescribing in itself was not the primary aim of the pharmacy team [30]. Polypharmacy can be an additional barrier for initiating therapies that might still impact quality of life or reduce morbidity [31]. As a consequence, the total number of drugs was not reduced during hospital stay, as multiple therapies were discontinued but also a comparable number of drugs were initiated. CP recommendations were mainly aimed toward improving cardiovascular therapies (28.7\%), which were regularly used on admission (42\%). The high prevalence of atrial fibrillation (44.5\%) was nearly identical to the findings of Tavernier et al., who found a prevalence of $46 \%$ in a comparable geriatric inpatient cohort [32]. Typical recommendations were to promote initiation of anticoagulation, or to use the appropriate dose of a new oral anticoagulant as validated in the landmark trials.

Our findings can be considered valid and reliable. First, EHR data were collected independently by two investigators and audited by a third afterwards. Data acquisition was nearly complete with only few missing data; full data from 519 out of 524 patients were available for analysis. Second, pharmacotherapeutic recommendations were prospectively documented by the CP team in a coded manner, using structured forms linked to the patient's EHR. Third, the analysis used data collected on an intervention provided in a mostly secondary health-care setting. Most patients admitted to the geriatric wards were living in the vicinity of the hospital. We hence assume that our results are generalizable to other hospitals and CP programs in older inpatients. Fourth, retained explanatory variables were biologically plausible.

Several important limitations of this study should be acknowledged, however. First, it concerned a retrospective study, using EHR-derived information. Second, an inherent bias could have been present in the CP team, unconsciously selecting patients with a higher pretest risk for drug-related issues (such as heart failure). This effect is however expected to be limited as the flow of the CP program did not include selection criteria. Third, due to the limited sample size of the cohort, the full cohort was not split into a training and validation set. Model performance was hence preliminary evaluated. Fourth, it is important to bear in mind that the explorative model was the best possible fit for the data at hand. This should not be considered equal to a good fit per se. Nonetheless, its predictive properties showed it to be a potentially useful model. Fifth, the dependent model outcome was an surrogate outcome measure, not directly linked to clinical relevance. Conversely, acceptance rate was high and the outcome was specifically chosen to increase the effectiveness of the program, not to ascertain clinical relevance per se.

Taking into account the study findings as well as the abovementioned limitations, five lessons for future investigations can be drawn. First, a prospective cohort study should be performed to further reduce the bias inherent to retrospective study designs. Second, in an unselected study sample, we need to further investigate the content of the provided and accepted CP recommendations, to assess their clinical relevance and also to evaluate what should be communicated to prescribers through academic detailing. Third, the prediction model should then be validated in a new and larger random sample. Fourth, the overall body of evidence has been expanding, yet there still is no consistent evidence to support CP services in geriatric inpatients, nor is it expected that this issue will be addressed in ongoing clinical trials [14]. The MEDBRIDGE study is currently the largest randomized controlled trial (RCT) in this setting, but will not specifically target geriatric inpatients [33]. Importantly, given the coverage of about $50-60 \%$ of our total geriatric population, a RCT could still be performed in adjunct to the CP program, powered to detect a reduction of all-cause readmissions [14]. Bearing in mind the results of our regression analysis, we would preferentially target patients who are polymedicated on admission and have not been admitted previously to acute geriatric care wards in our hospital. Fifth, a metric should be implemented to systematically evaluate the program's clinical relevance. Pellegrin et al. have developed and tested a composite of several ICD codes, allowing post hoc identification of drug-related admissions [13]. In our current setting, this might be feasible as part of an interrupted time series (ITS) analysis, analogous to the approach that Pellegrin et al. used, taking into account the lag time of ICD coding in our hospital of approximately 1 year. Such an investigation would concern a retrospective analysis, by definition. In addition, an ITS analysis might also be done using all-cause readmissions to the hospital as clinical outcome.

\section{Conclusion}

Clinical pharmacists on the geriatric ward of a large teaching hospital provided a median of three 
recommendations at discharge. Most recommendations concerned deprescribing. Five independent factors were identified that were associated with the number of $\mathrm{CP}$ recommendations at discharge. This could allow for further patient stratification to increase the efficiency of the current approach.

\section{Acknowledgments}

The authors wish to acknowledge the undergraduate pharmacists Emma Hendricks and Lieselot Vannitsem who assisted in the acquisition of data.

\section{Contributions of authors}

Lorenz Van der Linden was involved in study design, in overseeing data acquisition, analysis, interpretation of data and providing the first draft of the manuscript. Silke Loyens and Julie Hias participated in data acquisition and analysis. Julie Hias, Isabel Spriet, Jos Tournoy, Karolien Walgraeve and Johan Flamaing were involved in the study design, the interpretation of the data and final analysis. All authors participated in the preparation of the manuscript.

\section{Disclosure statement}

No potential conflict of interest was reported by the authors.

\section{References}

[1] Christensen M, Lundh A. Medication review in hospitalised patients to reduce morbidity and mortality. Cochrane Database Syst Rev. 2016;2:Cd008986.

[2] Clyne B, Fitzgerald C, Quinlan A, et al. Interventions to address potentially inappropriate prescribing in community-dwelling older adults: a systematic review of randomized controlled trials. J Am Geriatr Soc. 2016;64(6):1210-1222.

[3] Petrovic M, Somers A, Onder G. Optimization of geriatric pharmacotherapy: role of multifaceted cooperation in the hospital setting. Drugs Aging. 2016;33 (3):179-188.

[4] Spinewine A, Fialova D, Byrne S. The role of the pharmacist in optimizing pharmacotherapy in older people. Drugs Aging. 2012;29(6):495-510.

[5] Gray SL, Hart LA, Perera S, et al. Meta-analysis of interventions to reduce adverse drug reactions in older adults. J Am Geriatr Soc. 2018;66(2):282-288.

[6] Scullin C, Scott MG, Hogg A, et al. An innovative approach to integrated medicines management. J Eval Clin Pract. 2007;13(5):781-788.

[7] Johansen JS, Havnes K, Halvorsen KH, et al. Interdisciplinary collaboration across secondary and primary care to improve medication safety in the elderly (IMMENSE study): study protocol for a randomised controlled trial. BMJ Open. 2018;8(1):e020106.
[8] Hellstrom LM, Bondesson A, Hoglund P, et al. Impact of the Lund Integrated Medicines Management (LIMM) model on medication appropriateness and drug-related hospital revisits. Eur J Clin Pharmacol. 2011;67(7):741-752.

[9] Renaudin P, Boyer L, Esteve MA, et al. Do pharmacist-led medication reviews in hospitals help reduce hospital readmissions? A systematic review and meta-analysis. Br J Clin Pharmacol. 2016;82 (6):1660-1673.

[10] Ravn-Nielsen LV, Duckert ML, Lund ML, et al. Effect of an in-hospital multifaceted clinical pharmacist intervention on the risk of readmission: a randomized clinical trial. J JAMA Int Med. 2018;178(3):375-382.

[11] Van der Linden L, Decoutere L, Walgraeve K, et al. Combined use of the rationalization of home medication by an adjusted STOPP in older patients (RASP) list and a pharmacist-led medication review in very old inpatients: impact on quality of prescribing and clinical outcome. Drugs Aging. 2017;34(2):123-133.

[12] Walgraeve K, Van der Linden L, Flamaing J, et al. Feasibility of optimizing pharmacotherapy in heart failure patients admitted to an acute geriatric ward: role of the clinical pharmacist. Eur Geriatric Med. 2018;9(1):103-111.

[13] Pellegrin KL, Krenk L, Oakes SJ, et al. Reductions in medication-related hospitalizations in older adults with medication management by hospital and community pharmacists: a quasi-experimental study. J Am Geriatr Soc. 2017;65(1):212-219.

[14] Van der Linden L, Hias J, Walgraeve K, et al. Clinical pharmacy services on geriatric care wards: catch 22 of implementation and research. Drugs Aging. 2018;35 (5):375-377.

[15] Somers A, Spinewine A, Spriet I, et al. Development of clinical pharmacy in Belgian hospitals through pilot projects funded by the government. Acta Clin Belg. 2019;74(2):75-81.

[16] De Winter S, Vanbrabant $P$, Spriet I, et al. A simple tool to improve medication reconciliation at the emergency department. Eur J Intern Med. 2011;22(4):382-385.

[17] Van der Linden L, Hias J, Spriet I, et al. Medication review in older adults: importance of time to benefit. Am J Health Syst Pharm. 2019;76(4):247-250.

[18] American Geriatrics Society Expert Panel on the Care of Older Adults with Multimorbidity. Guiding principles for the care of older adults with multimorbidity: an approach for clinicians. J Am Geriatr Soc. 2012;60 (10):E1-E25.

[19] Garfinkel D, Mangin D. Feasibility study of a systematic approach for discontinuation of multiple medications in older adults: addressing polypharmacy. Arch Intern Med. 2010;170(18):1648-1654.

[20] Braekhus A, Laake K, Engedal K. The mini-mental state examination: identifying the most efficient variables for detecting cognitive impairment in the elderly. J Am Geriatr Soc. 1992;40(11):1139-1145.

[21] Jaeschke R, Guyatt GH, Sackett DL. Users' guides to the medical literature. III. How to use an article about a diagnostic test. B. What are the results and will they help me in caring for my patients? The evidence-based medicine working group. JAMA. 1994;271(9):703-707.

[22] El Hajji FW, Scullin C, Scott MG, et al. Enhanced clinical pharmacy service targeting tools: risk-predictive algorithms. J Eval Clin Pract. 2015;21(2):187-197.

[23] Falconer N, Barras M, Cottrell N. Systematic review of predictive risk models for adverse drug events in 
hospitalized patients. Br J Clin Pharmacol. 2018;84 (5):846-864.

[24] Ponikowski P, Voors AA, Anker SD, et al. ESC guidelines for the diagnosis and treatment of acute and chronic heart failure: the task force for the diagnosis and treatment of acute and chronic heart failure of the European society of cardiology (ESC). Developed with the special contribution of the Heart Failure Association (HFA) of the ESC. Eur J Heart Fail. 2016;18 (8):891-975.

[25] Lee SJ, Kim CM. Individualizing prevention for older adults. J Am Geriatr Soc. 2018;66(2):229-234.

[26] Somers A, Robays H, De Paepe P, et al. Evaluation of clinical pharmacist recommendations in the geriatric ward of a Belgian university hospital. Clin Interv Aging. 2013;8:703-709.

[27] De Bock L, Tommelein E, Baekelandt $H$, et al. The introduction of a full medication review process in a local hospital: successes and barriers of a pilot project in the geriatric ward. Pharmacy (Basel). 2018;6 (1):21.

[28] Somers A, Claus B, Vandewoude K, et al. Experience with the implementation of clinical pharmacy services and processes in a university hospital in Belgium. Drugs Aging. 2016;33(3):189-197.
[29] Ryan C, Ross S, Davey P, et al. Prevalence and causes of prescribing errors: the PRescribing outcomes for trainee doctors engaged in clinical training (PROTECT) study. PLoS One. 2014;9(1):e79802.

[30] Scott IA, Hilmer SN, Reeve E, et al. Reducing inappropriate polypharmacy: the process of deprescribing. JAMA Intern Med. 2015;175(5):827-834.

[31] Wauters $M$, Elseviers $M$, Vaes $B$, et al. Too many, too few, or too unsafe? Impact of inappropriate prescribing on mortality, and hospitalization in a cohort of community-dwelling oldest old. Br J Clin Pharmacol. 2016;82(5):1382-1392.

[32] Tavernier R, Wolf M, Kataria V, et al. Screening for atrial fibrillation in hospitalised geriatric patients. Heart. 2018;104(7):588-593.

[33] Kempen $\mathrm{TGH}$, Bertilsson $\mathrm{M}$, Lindner $\mathrm{KJ}$, et al. Medication reviews bridging healthcare (MedBridge): study protocol for a pragmatic cluster-randomised crossover trial. Contemp Clin Trials. 2017;61:126-132.

[34] Cockcroft DW, Gault MH. Prediction of creatinine clearance from serum creatinine. Nephron. 1976;16 (1):31-41.

[35] Levey AS, Stevens LA, Schmid CH, et al. A new equation to estimate glomerular filtration rate. Ann Intern Med. 2009;150(9):604-612. 